\title{
Logarithmic Sobolev Inequalities and Spectral Gaps
}

\author{
Eric Carlen ${ }^{1}$ and Michael Loss ${ }^{1}$ \\ School of Mathematics, Georgia Tech, Atlanta, GA 30332
}

January 24, 2004

\begin{abstract}
We prove an simple entropy inequality and apply it to the problem of determining logSobolev constants.
\end{abstract}

\section{Introduction}

Let $\mu$ be a probability measure on $\mathbb{R}^{n}$ of the form $\mathrm{d} \mu=e^{-V(x)} \mathrm{d} x$. One says that $\mu$ admits a logarithmic Sobolev inequality with constant $b$ in case for all functions $f$ on $\mathbb{R}^{n}$ with $\int_{R^{n}} f^{2} \mathrm{~d} \mu=1$,

$$
\int_{\mathbb{R}^{n}} f^{2} \ln f^{2} \mathrm{~d} \mu \leq b \int_{\mathbb{R}^{n}}|\nabla f|^{2} \mathrm{~d} \mu
$$

There is an extensive literature devoted to the specification of conditions under which there is a logarithmic Sobolev inequality associated to $\mu$, and the determination of the best constant $b$ when such an inequality does hold.

A number of years ago, it was shown by Bakry and Emery [2] that when $V$ is uniformly strictly convex; i.e., when the Hessian of $V$ satisfies

$$
\operatorname{Hess}_{V}(x) \geq 2 c I
$$

for all $x$, then (1.1) holds with $b=1 / c$. Since the method of Bakry and Emery requires that (1.2) hold uniformly, it does not apply in many cases of interest.

One says that $\mu$ admits a spectral gap with constant $\lambda$ in case for all functions $u$ on $\mathbb{R}^{n}$ with $\int_{\mathbb{R}^{n}} u \mathrm{~d} \mu=0$,

$$
\int_{\mathbb{R}^{n}} u^{2} \mathrm{~d} \mu \leq \frac{1}{\lambda} \int_{\mathbb{R}^{n}}|\nabla u|^{2} \mathrm{~d} \mu
$$

Taking $f$ to have the form $f=\sqrt{1-\alpha^{2}}+\alpha u$ where $u$ is orthogonal to the constants, a simple Taylor expansion yields

$$
\int_{\mathbb{R}^{n}} f^{2} \ln f^{2} \mathrm{~d} \mu=\alpha^{2}\left(3 \int_{\mathbb{R}^{n}} u^{2} \mathrm{~d} \mu-1\right)+\mathcal{O}\left(\alpha^{3}\right),
$$

\footnotetext{
${ }^{1}$ Work partially supported by U.S. National Science Foundation grant DMS 03-00349.

(C) 2004 by the authors. This paper may be reproduced, in its entirety, for non-commercial purposes.
} 
at least when $u$ is bounded. Since in this case we also have,

$$
\int_{\mathbb{R}^{n}}|\nabla f|^{2} \mathrm{~d} \mu=\alpha^{2} \int_{\mathbb{R}^{n}}|\nabla u|^{2} \mathrm{~d} \mu
$$

it follows that whenever $\mu$ admits a logarithmic Sobolev inequality with constant $b$, it admits a spectral gap with constant $\lambda \geq 2 / b$. This useful fact was observed by Rothaus [7].

Often it is much easier to prove a spectral gap than is is to prove a log-Sobolev inequality. Indeed, there are many examples of measures $\mu$ that admit a spectral gap, but do not admit a log-Sobolev inequality. At this meeting, several problems have been discussed in which a spectral gap has been proved, but a log-Sobolev inequality has not - or at least not with useful constants.

It can however be realtively easy to directly establish a restricted log-Sobolev inequality:

Definition In case for some finite $b,(1.1)$ is satisifed whenever

$$
\int_{\mathbb{R}^{n}} f \mathrm{~d} \mu=0 \quad \text { and } \quad \int_{\mathbb{R}^{n}} f^{2} \mathrm{~d} \mu=1
$$

them $\mu$ satisifes a restricted log-Sobolev inequality.

Our aim here is to show that whenever $\mu$ has the form $\mu=e^{-V(x)} \mathrm{d} x$, and $\mu$ admits a spectral gap, then under broad, easy to check conditions, $\mu$ admits a restricted log-Sobolev inequality with an explicit constant. ¿From this, we then deduce an unrestriced log-Sobolev constant for $\mu$. The passage from the restricted to the unrestricted inequality based on a simple $a-$ priori entropy inequality. We explain this in the second section, and then in the third section, we show how restricted log-Sobolev inequalities may be easily established.

The restriced log-Sobolev inequalities considered here are closely related to what are sometimes called deffective log-Sobolev inequalities. These take the form

$$
\int_{\mathbb{R}^{n}} f^{2} \ln f^{2} \mathrm{~d} \mu \leq b \int_{\mathbb{R}^{n}}|\nabla f|^{2} \mathrm{~d} \mu+a \int_{\mathbb{R}^{n}} f^{2} \mathrm{~d} \mu
$$

for all $f$ with $\int_{\mathbb{R}^{n}} f^{2} \mathrm{~d} \mu=1$. If $\mu$ admits a spectral gap with constant $\lambda$, and (1.6) is satisfied, then whenever $u$ satisifies (1.5),

$$
b \int_{\mathbb{R}^{n}}|\nabla u|^{2} \mathrm{~d} \mu+a \int_{\mathbb{R}^{n}} u^{2} \mathrm{~d} \mu \leq\left(b+\frac{a}{\lambda}\right) \int_{\mathbb{R}^{n}}|\nabla u|^{2} \mathrm{~d} \mu
$$

so that a defective log-Sobolev inequality, together with a spectral gap, implies a restricted logSobolev inequality.

There have been many investigations of log-Sobolev inequalities in the setting of diffusion semigroups, starting from the ground breaking work of Gross [6] demonstrating the equivalence of hypercontractivity of a diffusion semigroup with the validity of a log-Sobolev inequality for the associated Dirichlet form. See [1] for an insightful recent survey, and of particular relevance here, recent work of Cattiaux [3].

In particular, Cattiaux [3] has recently obtained log-Sobolev inequalities under conditions similar to those in Theorem 2.2 below, however his methods are considerably more complicated, and do not provide explicit constants. 
Many earlier researchers have also relied on diffusion semigruop arguments. For example, it is well known that a defective log-Sobolev inequality together with a spectral gap implies a logSobolev inequality. The standard proof uses Gross's Theorem and an argument of Glimm [5]. Gross's Theorem assures that if $\mu$ satisfies a defective log-Sobolev inequality, then the diffusion semigroup $P_{t}$ associated to the Dirichelt form $\int_{\mathbb{R}^{n}}|\nabla u|^{2} \mathrm{~d} \mu$ is bounded from $L^{2}$ to $L^{4}$ for some $t_{0}>0$. Next, the argument of Glimm is used to show that if a diffusion semigroup $P_{t}$ is bounded from $L^{2}$ to $L^{4}$ and some time $t_{0}$, and its generator has a spectral gap, then for some $t_{1}>t_{0}, P_{t_{1}}$ is a contraction from $L^{2}$ to $L^{4}$. This contractivity, together with an interpolation argument and Gross's Theorem once again give the log-Sobolev inequality.

This sort of argument is well known, but rather indirect. Our aim here is to provide a simple, direct passage from defective log-Sobolev inequalities to log-Sobolev inequalities via spectral gaps, and also to provide a simple and direct criterion for the validity of defective log-Sobolev inequalities. Because the arguments are simple and direct, they lead to sharper, more explicit results in many cases.

\section{A convexity inequality for entropy}

Our goal in this section is to prove the following inequality:

2.1 THEOREM. Let $\mu$ be any probability measure on a sigma algebra $\mathcal{S}$ of subsets of some set $\Omega$. Let $u$ be any measurable real valued function with

$$
\int_{\Omega} u^{2} \mathrm{~d} \mu=1 \quad \text { and } \quad \int_{\Omega} u \mathrm{~d} \mu=0 .
$$

For all $\alpha$ with $0 \leq \alpha \leq 1$, define

$$
f(x)=\sqrt{1-\alpha^{2}}+\alpha u(x)
$$

Then

$$
\int_{\Omega} f^{2} \ln f^{2} \mathrm{~d} \mu \leq 2 \alpha^{2}+\alpha^{4}+\alpha^{2} \int_{\Omega} u^{2} \ln u^{2} \mathrm{~d} \mu
$$

Before proving the theorem, we make several remarks. First, because $u$ is normalized in $L^{2}$ and is orthogonal to the constants, $f$ is also normalized in $L^{2}$. That is, both $u^{2} \mathrm{~d} \mu$ and $f^{2} \mathrm{~d} \mu$ are probability measures.

Second, the Taylor expansion (1.4) shows that the constant 2 is the best possible constant multiplying $\alpha^{2}$ in (2.1), for if $u(x)= \pm 1$ for every almost every $x$, then the right hand side of $(2.1)$ reduces to $2 \alpha^{2}+\alpha^{4}$.

Third, it might seem natural to try and prove (2.1) by controlling the remainder terms in the Taylor expansion. However, extracting an estimate on the remainder involving only $\alpha$ and $\int_{\Omega} u^{2} \ln u^{2} \mathrm{~d} \mu$ does not seem to be straightforward. Instead, we first prove an $L^{p}$ inequality that is an identity at $p=2$. Differentiation in $p$ will yield Theorem 2.1 . The $L^{p}$ inequality is the following: 
2.2 THEOREM. Let $\mu$ be any probability measure on a sigma algebra $\mathcal{S}$ of subsets of some set $\Omega$. Let $u$ be any measurable real valued function with

$$
\int_{\Omega} u^{2} \mathrm{~d} \mu=1 \quad \text { and } \quad \int_{\Omega} u \mathrm{~d} \mu=0 .
$$

For all $\alpha$ with $0 \leq \alpha \leq 1$, define

$$
f(x)=\sqrt{1-\alpha^{2}}+\alpha u(x)
$$

Then for $p \geq 2$,

$$
\|f\|_{p}^{p} \leq\left(1-\alpha^{2}\right)^{p / 2}+\frac{p(p-1)}{2}\|f\|_{p}^{(p-2)} \alpha^{2}\|u\|_{p}^{2} .
$$

Proof: For $t$ real, define $\phi(t)$ by

$$
\phi(t)=\int_{\Omega}|c+t u|^{p} \mathrm{~d} \mu
$$

where $c=\sqrt{1-\alpha^{2}}$. Differentiating, we find

$$
\phi^{\prime}(t)=p \int_{\Omega}\left((c+t u)^{2}\right)^{p / 2-1}(c+t u) u \mathrm{~d} \mu .
$$

In particular,

$$
\phi^{\prime}(0)=p|c|^{p-2} c \int_{\Omega} u \mathrm{~d} \mu=0 .
$$

Differentiating once more, we find

$$
\phi^{\prime \prime}(t)=p(p-1) \int_{\Omega}|c+t u|^{p-2} u^{2} \mathrm{~d} \mu .
$$

Applying Holder's inequality with indices $p /(p-2)$ and $p / 2$, we obtain

$$
\begin{aligned}
\phi^{\prime \prime}(t) & \leq p(p-1)\|c+t u\|_{p}^{p-2}\|u\|_{p}^{2} \\
& =p(p-1) \phi(t)^{(p-2) / p}\|u\|_{p}^{2} .
\end{aligned}
$$

Together, (2.3) and (2.4) show that $\phi$ is increasing in $t \geq 0$, and thus, for all $t$ with $0 \leq t \leq \alpha$, we have from (2.5) that

$$
\phi^{\prime \prime}(t) \leq p(p-1) \phi(\alpha)^{(p-2) / p}\|u\|_{p}^{2} .
$$

Then, once again using (2.3),

$$
\phi(\alpha)=\phi(0)+\int_{0}^{\alpha} \int_{0}^{t} \phi^{\prime \prime}(s) \mathrm{d} s \mathrm{~d} t .
$$

Using the estimate (2.6) in (2.7) yields the result.

Proof of Theorem 2.1: We notice first that (2.2) holds as an equality at $p=2$. We may therefore differentiate both sides in $p$ at $p=2$ to obtain a new inequality. We first compute

$$
\frac{\mathrm{d}}{\mathrm{d} p}\|f\|_{p}^{p-2}=\left[\frac{2}{p^{2}} \ln \left(\int_{\Omega}|f|^{p} \mathrm{~d} \mu\right)-\frac{p-2}{p} \frac{1}{\|f\|_{p}^{p}}\left(\int_{\Omega}|f|^{p} \ln |f| \mathrm{d} \mu\right)\right]\|f\|_{p}^{p-2} .
$$


Since $\|f\|_{2}=1$, this vanishes at $p=2$.

Next, for $g=\alpha u$,

$$
\frac{\mathrm{d}}{\mathrm{d} p}\|g\|_{p}^{2}=\left[-\frac{2}{p^{2}} \ln \|g\|_{p}^{p}+\frac{2}{p} \frac{1}{\|g\|_{p}^{p}}\left(\int_{\Omega}|g|^{p} \ln |g| \mathrm{d} \mu\right)\right] .
$$

At $p=2$, this reduces to

$$
\frac{1}{2} \int_{\Omega} g^{2} \ln \left(\frac{g^{2}}{\|g\|_{2}^{2}}\right) \mathrm{d} \mu=\frac{\alpha^{2}}{2} \int_{\Omega} u^{2} \ln u^{2} \mathrm{~d} \mu .
$$

Finally, the derivative of $p(p-1) / 2$ at $p=2$ is $3 / 2$, and the derivative of $c^{p}$ at $p=2$ is $c^{p} \ln c$.

On the left hand side, the derivative of $\|f\|_{p}^{p}$ at $p=2$ is $\int_{\Omega} f^{2} \ln |f| \mathrm{d} \mu$. Altogether, we have

$$
\int_{\Omega} f^{2} \ln f^{2} \mathrm{~d} \mu \leq\left(1-\alpha^{2}\right) \ln \left(1-\alpha^{2}\right)+3 \alpha^{2}+\alpha^{2} \int_{\Omega} u^{2} \ln u^{2} \mathrm{~d} \mu .
$$

However, by concavity of the logarithm,

$$
\left(1-\alpha^{2}\right) \ln \left(1-\alpha^{2}\right) \leq-\left(1-\alpha^{2}\right) \alpha^{2}
$$

so that

$$
\left(1-\alpha^{2}\right) \ln \left(1-\alpha^{2}\right)+3 \alpha^{2} \leq 2 \alpha^{2}+\alpha^{4} .
$$

Using this in (2.8) gives us (2.1).

\section{Application to logarithmic Sobolev inequalities}

In this section, we consider $\Omega=\mathbb{R}^{n}$, and

$$
\mathrm{d} \mu=e^{-V(x)} \mathrm{d}^{n} x,
$$

and are concerned with the following question:

Suppose $V$ is such that $\mu$ admits a spectral gap with constant $\lambda$. What further conditions on $V$ then ensure that $\mu$ also admits a logarithmic Sobolev constant for some finite constant $b$ ?

The following lemmas provide a positive answer. The first says that if $\mu$ admits a spectral gap, and if $\mu$ admits a restricted log-Sobolev inequality, then it admits an unrestriced log-Sobolev inequality, and it provides a simple estimate for the constant.

3.1 LEMMA. Suppose that $\mu$ admits a spectral gap $\lambda>0$, and for some finite $b$,

$$
\int_{\mathbb{R}^{n}} u^{2} \ln u^{2} \mathrm{~d} \mu \leq b \int_{\mathbb{R}^{n}}|\nabla u|^{2} \mathrm{~d} \mu
$$

whenever

$$
\int_{\mathbb{R}^{n}} u \mathrm{~d} \mu=0 \quad \text { and } \quad \int_{\mathbb{R}^{n}} u^{2} \mathrm{~d} \mu=1 .
$$

Then $\mu$ admits a logarithmic Sobolev inequality with constant no larger than

$$
b+\frac{3}{\lambda}
$$


Proof: Consider any $f$ with $\int_{\mathbb{R}^{n}} f^{2} \mathrm{~d} \mu=1$, and write it in the form considered in Theorem 2.1. Then, by Theorem 2.1,

$$
\int_{\mathbb{R}^{n}} f^{2} \ln f^{2} \mathrm{~d} \mu \leq 3 \alpha^{2}+\alpha^{2} \int_{\mathbb{R}^{n}} u^{2} \ln u^{2} \mathrm{~d} \mu
$$

By the spectral gap inequality,

$$
\alpha^{2}=\alpha^{2} \int_{\mathbb{R}^{n}} u^{2} \mathrm{~d} \mu \leq \frac{\alpha^{2}}{\lambda} \int_{\mathbb{R}^{n}}|\nabla u|^{2} \mathrm{~d} \mu \leq \frac{1}{\lambda} \int_{\mathbb{R}^{n}}|\nabla f|^{2} \mathrm{~d} \mu
$$

By hypothesis

$$
\alpha^{2} \int_{\mathbb{R}^{n}} u^{2} \ln u^{2} \mathrm{~d} \mu \leq b \alpha^{2} \int_{\mathbb{R}^{n}}|\nabla u|^{2} \mathrm{~d} \mu \leq b \int_{\mathbb{R}^{n}}|\nabla f|^{2} \mathrm{~d} \mu .
$$

This yields the result.

The next lemma gives conditions under which a restricted log-Sobolev inequality may be proven.

3.2 LEMMA. Suppose that $V$ is $C^{2}$, and that $\mu$ admits a spectral gap $\lambda>0$, and

$$
-C=\inf _{x}\left\{\frac{1}{4}|\nabla V(x)|^{2}-\frac{1}{2} \Delta V(x)-\pi e^{2} V(x)\right\}>-\infty .
$$

Then for all u satisfying

$$
\begin{gathered}
\int_{\mathbb{R}^{n}} u \mathrm{~d} \mu=0 \quad \text { and } \quad \int_{\mathbb{R}^{n}} u^{2} \mathrm{~d} \mu=1, \\
\int_{\mathbb{R}^{n}} u^{2} \ln u^{2} \mathrm{~d} \mu \leq \frac{\lambda}{(\lambda+|C|) \pi e^{2}} \int_{\mathbb{R}^{n}}|\nabla u|^{2} \mathrm{~d} \mu .
\end{gathered}
$$

Before proving Lemma 3.2, we recall a special case of the family of logarithmic Sobolev inequalities on $\mathbb{R}^{n}$ equipped with Lebesgue measure: For all functions $g$ on $\mathbb{R}^{n}$ with $\int_{\mathbb{R}^{n}} g^{2} \mathrm{~d}^{n} x=1$,

$$
\int_{\mathbb{R}^{n}} g^{2} \ln g^{2} \mathrm{~d}^{n} x \leq \frac{1}{\pi e^{2}} \int_{\mathbb{R}^{n}}|\nabla g|^{2} \mathrm{~d}^{n} x
$$

Proof of Lemma 3.2: Consider any function $u$ with $\int_{\mathbb{R}^{n}} u \mathrm{~d} \mu=0$, and $\int_{\mathbb{R}^{n}} u^{2} \mathrm{~d} \mu=1$. Then for any $t$ with $0<t<1$,

$$
\begin{aligned}
\int_{\mathbb{R}^{n}}|\nabla u|^{2} \mathrm{~d} \mu & -t \pi e^{2} \int_{\mathbb{R}^{n}} u^{2} \ln u^{2} \mathrm{~d} \mu \\
& =(1-t) \int_{\mathbb{R}^{n}}|\nabla u|^{2} \mathrm{~d} \mu+t\left(\int_{\mathbb{R}^{n}}|\nabla u|^{2} \mathrm{~d} \mu-\pi e^{2} \int_{\mathbb{R}^{n}} u^{2} \ln u^{2} \mathrm{~d} \mu\right) \\
& \geq(1-t) \lambda \int_{\mathbb{R}^{n}} u^{2} \mathrm{~d} \mu+t\left(\int_{\mathbb{R}^{n}}|\nabla u|^{2} \mathrm{~d} \mu-\pi e^{2} \int_{\mathbb{R}^{n}} u^{2} \ln u^{2} \mathrm{~d} \mu\right) .
\end{aligned}
$$

Next, define

$$
g(x)=u(x) e^{-V(x) / 2}
$$

so that

$$
\int_{\mathbb{R}^{n}} u^{2} \mathrm{~d} \mu=\int_{\mathbb{R}^{n}} g^{2} \mathrm{~d}^{n} x .
$$


When $U$ is smooth with compact support and $V$, a simple computation reveals

$$
\int_{\mathbb{R}^{n}}|\nabla u|^{2} \mathrm{~d} \mu=\int_{\mathbb{R}^{n}}|\nabla g|^{2} \mathrm{~d}^{n} x+\int_{\mathbb{R}^{n}} W g^{2} \mathrm{~d}^{n} x
$$

where

$$
W(x)=\frac{1}{4}|\nabla V(x)|^{2}-\frac{1}{2} \Delta V(x) .
$$

A standard approximation argument shows identity, the so-called "ground state stransformation" is generally valid. An even simpler computation reveals

$$
\int_{\mathbb{R}^{n}} u^{2} \ln u^{2} \mathrm{~d} \mu=\int_{\mathbb{R}^{n}} g^{2} \ln g^{2} \mathrm{~d}^{n} x+\int_{\mathbb{R}^{n}} V g^{2} \mathrm{~d}^{n} x .
$$

Therefore,

$$
\begin{aligned}
\int_{\mathbb{R}^{n}}|\nabla u|^{2} \mathrm{~d} \mu & -\pi e^{2} \int_{\mathbb{R}^{n}} u^{2} \ln u^{2} \mathrm{~d} \mu \\
& =\int_{\mathbb{R}^{n}}|\nabla g|^{2} \mathrm{~d}^{n} x-\pi e^{2} \int_{\mathbb{R}^{n}} g^{2} \ln g^{2} \mathrm{~d}^{n} x \\
& +\int_{\mathbb{R}^{n}}\left(W-\pi e^{2} V\right) g^{2} \mathrm{~d}^{n} x \\
& \geq \int_{\mathbb{R}^{n}}\left(W-\pi e^{2} V\right) g^{2} \mathrm{~d}^{n} x
\end{aligned}
$$

Therefore,

$$
\int_{\mathbb{R}^{n}}|\nabla u|^{2} \mathrm{~d} \mu-t \pi e^{2} \int_{\mathbb{R}^{n}} u^{2} \ln u^{2} \mathrm{~d} \mu \geq \int_{\mathbb{R}^{n}}\left[(1-t) \lambda+t\left(W-\pi e^{2} V\right)\right] g^{2} \mathrm{~d}^{n} x .
$$

The integrand is non negative provided that

$$
\frac{1}{4}|\nabla V(x)|^{2}-\frac{1}{2} \Delta V(x)-\pi e^{2} V(x)+\frac{1-t}{t} \lambda \geq 0
$$

for all $x$. Define $C$ by (3.1).

Then provided $C$ is finite, we can chose $t=\lambda /(\lambda+|C|)$, and the integrand will be positive.

Lemmas 3.1 and 3.2 immediately yield the following theorem:

3.3 THEOREM. Suppose that $V$ is $C^{2}$ and that $\mu$ admits a spectral gap $\lambda>0$, and

$$
-C=\inf _{x}\left\{\frac{1}{4}|\nabla V(x)|^{2}-\frac{1}{2} \Delta V(x)-\pi e^{2} V(x)\right\}>-\infty .
$$

then $\mu$ admits a logarithmic Sobolev inequality with constant $b$ no larger than

$$
\frac{\lambda}{(\lambda+|C|) \pi e^{2}}+\frac{3}{\lambda}
$$

Notice that if $V(x) \asymp|x|^{\gamma}$ and $|\nabla V(x)| \asymp|x|^{\gamma-1}$, then the condition that $C$ is finite requires $\gamma \geq 2$. This is consistent with the fact that whenever $\mu$ admits a logarithmic Sobolev inequality with some finite constant $b$, there is a number $\beta>0$ so that

$$
\int_{\mathbb{R}^{n}} e^{\beta|x|^{2}} \mathrm{~d} \mu<\infty
$$


Thus, concerning qualitative growth conditions on $V$, Theorem 3.3 is sharp. It is however surprising that the Laplacian of $V$ enters $C$ with a negative sign, given that the Bakry-Emery condition implies a logarithmic Sobolev inequality for $\mu$ whenever the Hessian of $V$ is uniformly bounded below.

To end the discussion, let us note that in one dimension estimates of the gap are relatively easy to come by. It was shown before that with $u=e^{V / 2} g(1.3)$ reduces to

$$
\int_{\mathbb{R}^{n}}|\nabla g(x)|^{2}+\left[\frac{|\nabla V|^{2}}{4}-\frac{\Delta V}{2}\right]|g|^{2} \mathrm{~d}^{n} x \geq \lambda \int_{\mathbb{R}^{n}}|g|^{2} \mathrm{~d}^{n} x .
$$

and must hold for all functions $g$ satisfying the condition $\int_{\mathbb{R}^{n}} g e^{-V / 2} \mathrm{~d}^{n} x=0$. In other words, the best possible value for $\lambda$ is given by the gap of the Schrödinger operator

$$
-\Delta g+\left[\frac{|\nabla V|^{2}}{4}-\frac{\Delta V}{2}\right] g=\lambda g .
$$

Clearly, the function $g_{0}=e^{-V / 2}$ is the ground state of this Schrödinger equation with corresponding eigenvalue zero. By an elementary calculation is easily seen that $\lambda$ is the second eigenvalue of the operator (on $\left.L^{2}(\mathbb{R}, \mathrm{d} x)\right)$

$$
\left(-\frac{\mathrm{d}}{\mathrm{d} x}-\frac{g_{0}^{\prime}}{g_{0}}\right)\left(\frac{\mathrm{d}}{\mathrm{d} x}-\frac{g_{0}^{\prime}}{g_{0}}\right)
$$

By the well known commutation formula (see, e.g. [4] ), the operator

$$
\left(\frac{\mathrm{d}}{\mathrm{d} x}-\frac{g_{0}^{\prime}}{g_{0}}\right)\left(-\frac{\mathrm{d}}{\mathrm{d} x}-\frac{g_{0}^{\prime}}{g_{0}}\right)
$$

has $\lambda$ as its lowest eigenvalue, in fact it has the same spectrum as (3.3) except for the lowest eiegnvalue zero. Thus, the gap $\lambda$ is now the lowest eigenvalue of the operator

$$
\frac{-\mathrm{d}^{2}}{\mathrm{~d} x^{2}}+\frac{V^{\prime \prime}}{2}+\frac{V^{\prime 2}}{4}
$$

which is given by an unconstrained minimization. Notice also, that here the second derivative of the potential shows up with the "right" sign.

\section{Acknowledgements}

This paper grew out of discussions betwen the authors while both were visiting Cedric Villani at E.N.S. Lyon in June 2003. We thank Cedric for hosting us, and for many interesting discussion on a problem of proving a family of log-Sobolev inequalities on $\mathbb{R}^{n}$ with constants independent of the dimension. This problem arises in a large deviations problem considered by several authors, and in particular, Otto and Villani, and is explained in these procedings.

\section{References}

[1] C. Ane et al., Sur les inégalités de Sobolev Logarithmiques, Soc. Math de France, Panoramas et Synthèses, No. 10, 200. 
[2] D. Bakry, M. Emery Hypercontractivité de semi-groups de diffusion, C. R. Acad. Sci. Paris Sér I Math. 299 775-778 (1984)

[3] P. Cattiaux, Hypercontractivity for perturbed diffusion semigroups, preprint, 2003.

[4] P.A. Deift, Applications of a commutation formula, Duke Math. J. 45 267-310 (1978).

[5] J. Glimm, Boson fields with nonlinear self interaction in two dimensions, Commun. Math. Phys., 8 12-25 (1968)

[6] L. Gross, Logarithmic Sobolev inequalities, Amer. Jour. Math, 97 1061-1083 (1976)

[7] O.S. Rothaus, Lower bounds for eigenvalues of regular Sturm-Liouville operators and the logarithmic Sobolev inequality, Duke Math. Jour. 45 351-362 (1978) 